einer aus dem Salzlager der Mecklenburgischen Gewerkschaft Friedrich Franz zu Lübtheen stammenden Lauge, die durch ihren Gehalt an L i t h i u m als "Urlauge" charakterisiert war ${ }^{5}$ ), zu 2,7 mg im Liter feststellte (gewogen als Palladiumjodür).

W i n $\mathbf{k}$ l e r hat also lediglich die bereits bekannte Tatsache bestätigt, daß Urlaugen verhältnismäßig reich an Jod sind, so daß man es darin auch mit einer weniger vollkommenen, aber schneller ausführbaren Methode auffinden und colorimetrisch bestimmen kann.

[A. 16.]

\section{Der Ausschluß der öffentlichkeit für Patente und Gebrauchsmuster.}

\author{
Von Dr. Julios Ephraim, Patentanwalt.
} (Fingeg. $22 / 221917$.

Der Bundesrat hat am 8./2. 1917 eine Bekanntmachung über den Ausschluß der Öffentlichkeit für Patente und Gebrauchsmuster erJassen ${ }^{1}$ ). Durch diese Verordnung, die mit dem Tage der Verkündung also am 8./2. 1917, in Kraft getreten ist und bis zur Bestimmung des Zeitpunktes für das Außerkrafttreten durch den Reichskanzler in Wirksamkeit bleibt, ist eine grundsätzliche Änderung des Patentund Gebrauchsmustergesetzes eingetreten. Diese Änderung dürfte namentlich für die chemische Industrie Bedeutung haben, da de AusschIuß der Öffentlichkeit sich auf Erfindungen im Interesse der Landesverteidigung und der Kriegswirtschaft bezieht, und gerade die chemische Industrie für die Landesverteidigung und Kriegswirtschaft in erster Linie mit in Betracht kommt.

Es gab bereits nach dem goltenden Patentgesetze Geheimpatente. Dieselben betrafen aber lediglich Erfindungen, die in Namen der Reichsverwaltung für die Zwecke des Heeres oder der Flotte angemeldet waren ( $\$ 19$, Absatz 3 des Patentgesetzes), während eine Geheimhaltung von Patenten, die nicht im Namen des Reichs, sondern seitens privater Stellen angemeldet waren, nicht vorgesehen war. Diese Verhältnisse werden nunmehr geändert.

Nach der Bekanntmachung findet die Erteilung eines Patentes ohno jede Bekanntmachung statt, wenn das Patentamt nach Anhörung der Heeres- und der Marineverwaltung die Geheimhaltung der Erfindung im Interessz der Landesverteidigung oder der Kriegswirtschaft für erforderlich erachtet. Entsprechendes gilt für die Eintragung eines Gebrauchsmusters (Bekanntmachung \$ 1, Absatz 1 und 2). Über das Verfahren bei der Erteilung derartiger Patente ist in der Bekanntmachung nichts ausgeführt. Die Regelung des Verfahrens ergibt sich nach den allgemeinen Grundsätzen des Patentgesetzes.

Das Patentamt entscheidet von sich aus, ob die Geheimhaltung der Erfindung erforderlich ist. Das Patentamt kann diese Entseheidung ohne äußere Anregung treffen. Fs ist aber natülich nicht ausgeschlossen, da B der Anmelder entweder gleich bei der Anmelduns oder im Laufe des Vorpröfungsverfahrens den Antrag auf Erteilung eines Gehcimpatentes stellt. In jedem Falle ist die Anhörung der Heeres- und der Mrrineverwaltung notwendig. Die Bekanntgabe des Ergebnissıs der Anhörung der Hoeres- und Marineverwaltung an den Anmolder wird unterbleiben. Allerdings sagt hierüber die Bekanntmachung nichts, doch ist mit Rücksicht auf den zu erwartenden Inhalt der Äußarung der Heeres- und der Marineverwaltung anzunehmen, da $\beta$ dis Geheimhaltung der Äußerung notwendig sein dürfte. Unter der Anhörung ist nach der Ausdrucksweise des Patentgesetzes eine mündliche Erörterung zu verstehen. Es würde abcr zweifellos nichts im Wege sein, daß für das Verfahren zur Ausführung der Be kanntmachung der schriftliche Weg eingeschlagen wird. Das Patentamt ist an die Äußerung der Heeres- und der Marineverwaltung nicht gebunden, da die Entscheidung über die Erteilung des Geheimpatentes lediglich beim Patentamte liegt, und die Bestimmung ïber die Anhörung nur bedeutet, daß eine Meinungsäußerung eingeholt werden soll. Grundsätzlich ist es nicht ausgeschlossen, da $B$ das Patentamt eine Entscheidung im Gegensatz zu der Meinungsäußerung der Heres- und Marineverwaltung trifft. Die Hecres- und Marineverwal tung kann der Ansicht sein, da 3 eine Geheimhaltung nicht erforderlich ist, währınd das Patentamt, trotz dieser Auffassung der Behörden, die Geheimhaltung für notwendig erachtet. Wenngleich die Geheimhaltung nach dem Wortlaute der Bekanntmachung nur dann statt-

5) Nach W. Feit, vgl. E. Erdmann, Die Chemie und Industrie der Kalisalze (S. 27), al:s „Deutschlands Kalibergbau“, Berlin 1907 (Verlag der Königl. Geclog. Landesanstalt)

1) Vgl. Angew. Chem. 30, III, 105 [1917]. finden soll, wenn die Geheimhaltung „erforderlich" ist, so ist die Bestimmung nicht derartig aufzufassen, daß es sich um eine unbedingt notwendige Geheimhaltung handelt, vielmehr kann auch die Erteilung des Geheimpatentes stattfinden, wenn das Patentamt zu der Ansicht gelangt, daß eine Geheimhaltung immerhin nützlich sein würde.

Im Falle, da $B$ der Patentanmelder seinerseits die Geheimhaltung für notwendig erachtet, wird es empfehlenswert sein, sich nicht auf einen einfachen Antrag zu beschränken, vielmehr wird es sich empfehlen, die Gründe näher darzulegen, aus denen nach Anschauung des Anmelders die Geheimhaltung erforderlich ist.

Wenn das Patentamt die Erteilung eines Geheimpatentes für notwendig erachtet, so fällt die Bekanntmachung der Anmeldung fort. Der Beschluß, ein Geheimpatent zu erteilen, muß also vor der Ausführung der Bekanntmachung stattfinden, dagegen würde es nicht unzulässig sein, wenn nach Fassung des Bekanntmachungsbeschlusses vor der tatsächlichen Ausführung desselben die Erteilung des Gcheimpatentes stattfindet.

Der Beschluß, ein Geheimpatent zu erteilen, ist als einheitlicher Beschluß anzusehen, der sowohl die Erteilung eines Patentes, wic den Geheimcharakter dieses Patentes in sich einheitlich umfaßt. Wenn das Patentamt ein Geheimpatent erteilt, so kann der Anmelder nicht ausschlieBlich dagegen die Beschwerde einlegen, da $B$ das erteilte Patent geheimgehalten werden soll, dagegen der Erteilung eines Patentes zustimmen.

Wenn der Anmelder damit nicht einverstanden ist, da $B$ das erteilte Patent geheimgehalten werden soll, so kann er die Beschwcrde einlegen, muß aber damit rechnen, duß die Beschwerdeabteilung auch die Frage nachprüft, ob überhaupt ein Patent erteilt werden soll. Fraglich kann die Art des Beschwerdeweges sein. Da im $\$ 26 \mathrm{des}$ Patentgesetzes bestimmt ist, da $B$ der Patentsucher gegen den Beschluß, durch welchen über die Erteilung des Patentes entschieden wird, unter Zahlung einer Gebühr von $20 \mathrm{M}$. innerhalb cines MIonats nach der Zustellung des Beschlusses Beschwcrde einlegen kann, so wird anzunehmen sein, daß die Beschwerde gegen die Erteilung des Geheimpatentes sich auch nach dieser Bestimmung richtet. Es würde also die zahlungspflichtige Beschwcrde innerhalb eines Monates einzulegen sein und nicht die frist- und kostenlose Beschwerde nach $\$ 16$ des Patentgesctzes. Die Notwendigkeit, die Beschwe rde inrerhalb einer Frist einzureichen, ergibt sich auch daraus, daB die Beschwerde vor Ausführung des Beschlusses über die Erteilung c'es Geheimpatentes, also vor Eintragung des Patentes in die Kriegsrcile erhoben sein muß.

Die vor:tehende Auffassung is t aus dem Wortlaute der Eckanntmachung abceleitet. Die Begı ündung zu dcrselken vertritt die Ansicht, daß d:e Ertoilung e res Gele:mpatentes nur mit $\mathrm{Zu}$ stimmung c'es Anne'rers erfolcen kann, während ke:m Fch en derse'ten die Bckanntmachung der Anr e'dung ausgesc tzt wird. D ese Zurückhaltung der Eekanntmachın's st ht aber im Ge censatze zum Pat. nt e etre und erfolcte nur, cewssermaßen im W derspruclie zum Gesetze, unter dem Zwange c'er Verhältnis se. Die vorliecende Bckanntmachung soll eine ces: tzliche Rer olung r'es Vorgcliens lec'outen, so daß damit die bisl erige Praxs auler Kraft gcs etzt würde. De in der Begründung zum Gı setze entlialtene Auffassung über das Verfahren steht nach meiner Auffassung im Widerspruch zum Wortlaut der Pekanntmachung und hätte in diesem Falle keine Geltung. Allerdings wird das Patentamt wahrscheinlich der Eegründung folgen.

Im Falle, daß der Antrag auf Erteilung cines Geheimpatentes seitens des Patentamtes abgelehnt werden sollte, hat der Anmelde gleichfalls das Beschwerderecht. Auch diese Beschwerde wircl sich nach den Bestimmungen des $\$ 26$ des Patentgesetzes zu richtrn haben und nicht nach $\$ 16$, denn die Ablehnung des Antrages, cin Geheimpatent zu erteilen, bedeutet einen Beschluß über die Erteilung des Patentes.

Die Marine- und Heeresverwaltung ist an dem Verfahren der Patenterteilung nicht als Partei beteiligt. Infolgedescen kann von dieser Stelle aus keine Beschwerde eingelegt werden, falls das $\mathrm{Pa}$ tentamt im Widerspruch zu der Meinungsäußerung der Hecres- und Marineverwaltung entschieden haben sollte.

Im Falle eines Gebrauchsmusters besteht cin frist- und kostenloses Beschwerdeverfahren, das im wesentlichen cine Vorstellung an den Präsidenten des Patentamtes darstellt.

Das Geheimpatent wird in einen besonderen geheimen Band der Patent- oder Gebrauchsmusterrolle eingetragen (Kriegsrolle). Der Inhalt der Kriegsrolle wird nicht veröffentlicht. Es wird also weder die E te lung eines Patentes, noch der Name des Patentinhabers veröffentlicht. 
Da bei dem Geheimpatente die Bekanntmachung der Anmeldung und ihre Auslegung unterbleibt, fällt auch der Bekanntmachungsbeschluß fort. Hiernach wird die Verzögerung des Erteilungsverfahrens, die durch die Aussetzung der Bekanntmachung um 3 bzw. 6 Monate (\$23 Absatz 4 des Patentgesetzes) möglich ist, fortfallen. Dies kann für den Patentinhaber bei der Nachsuchung von Auslandspatenten von Bedeutung sein. Nach dem Patentgesetze der Vereinigten Staaten muß die Anmeldung eines Patentes innerhalb 12 Monaten nach Anmeldung des betreffenden Auslandspatentes erfolgen, wenn das Auslandspatent vor dem amerikanischen Patent zur Erteilung gelangt. Im anderen Falle kann kein rochtsgültiges amerikanisches Patent erteilt werden. Die rechtzeitige Einreichung eines Patentes in den Vercinigten Staaten ist unter den jetzigen Verhältnissen in Frage gestellt. Die Verzögerung der Erteilung eines Patentes bei einem Geheimpatente ist aber nicht möglich, da wohl die Bekanntmachung der Anmeldung, nicht aber die Verzögerung der Erteilung eines Patentes nach dem geltenden Patentgesetze vorgesehen ist. Es ist wobl damit zu rechnen, $\mathrm{da} ß$ nach Beendigung des Krieges zwischen den einzelnen Staaten über die Beseitigung der im Krieg eingetretenen Hindernisse für die Errteilung rechtsgültiger Patente ein allgemeines Abkommen getroffen wird. Für dieses Abkommen wird gerade die Frage der durch die Erteilung von Geheimpatenten gesohaffenen Sachlage in Betracht zu ziehen sein.

Die Folge der Erteilung eines Geheimpatentes ist, daß die Einsicht der Kriegsrolle sowie der Anmeldestücke nicht gestattet ist. Der Heeres- und Marineverwaltung steht die Einsicht der Akten zu. Anderen Personen kann die Einsicht der Kriegsrolle sowie der Akten über die Geheimpatente und Geheimgebrauchsmuster auf Antrag mit Zustimmung der Heeres- und Marineverwaltung von dem $\mathrm{Pa}$ tentamte gestattet werden ( $\$ 2$ ). Die Wirkung der Erteilung eines Geheimpatentes (und -rebrauchsmusters) ist diejenige der gewöhnlichen Patente (und Gebrauchsmuster). Der Patentinhaber ist hiernach ausschließlich befugt, den Gegenstand der Erfindung gewerbsmäBig herzustellen, in Verkehr zu bringen, feil zu halten oder zu gebrauchen. Die unbefugte Benutzung der Erfindung durch andere Personen ist auch bei den Geheimpatenten verboten. Schwierigkeiten könnten bei der Verfolgung von Verletzungen der Geheimpatente daduroh entstehen, daß nur bei grober Fahrlässigkeit oder Wissentlichkeit der Verletzung Schadenersatzpflicht besteht, während Verletzungen, bei denen diese Voraussetzungen nicht gegeben sind, keinen $\mathbf{\Delta a}$ :pruch auf Schadenersatz begründen. $\mathrm{Da}$ nun eine der Voraussetzungen für grobe Fahrlässigkeit und Wissentlichkeit darin besteht, daß der Verletzer die Möglichkeit hat, sich über den Inhalt und Schutzumfang des erteilten Patentes zu unterrichten, diese Möglichkeit aber bei dem Geheimpatente fortfällt, kann es zunächst fraglich sein, ob Verletzungen der Geheimpatente zum Schadenersatz verpflichten. Die Rechtsprechung dürfte die hierdurch etwa entstehenden Schwierigkeiten aber leicht beheben. Nach der Sachlage des Falles wird das Gericht zu entscheiden haben, ob auch bei Nichtkennen des Patentes nicht grobe Fahrlässigkeit bestanden haben kann. In vielen Fällen wird der Verletzer, namentlich wenn ihm vom Bestehen eines Geheimpatentes Mitteilung gemacht wird, auch sich sagen können, welchen Inhalt dieses Patent hat. Wenn er trotzdem die Erfindung benutzt, so ist grobe Fahrlässigkeit deshalb gegeben, weil der Verletzer mit der Möglichkeit einer Verletzung rechnen muBte und unbckümmert um diese Möglichkeit an die Benutzung der Erfindung ging. Es bestände also ,dolus eventualis". Im Falle einer Verletzungsklage darf der Inhalt des Patentes einem Verletzer nicht mitgeteilt werden, falls nicht auf seinen Antrag ihm die Einsicht der Akten mit Zustimmung der Həeres- und Marineverwaltung gestattet wird. Ob diese Einsichtnahme in Falle eines anhängigen Verletzungsprozesses gswährt wird, hängt von der Entscheidung des Patentamtes ab. Wenn der Nachweis geführt wird, daB der Inhaber eines Geheimpatentes eine Klage wegen Verletzung anhängig gemacht hat, wird, falls die Heeres- und die Marineverwaltung zustimmt, die Einsichtnahme wohl gestattet werden. Das Patentamt kann die Einsichtnahme von bestimmten Bedingungen, welche einer Verbreitung des Inhaltes der Akten vorbeugen, abhängig machen. Die unbefugte Verbreitung der Kenntnis der Akten wird nach \$ 4 der Bekanntmachung übrigens mit Gefängnis bis zu einem Jahr und mit Geldstrafen bis zu $5000 \mathrm{M}$ oder mit einer dieser Strafen bestraft.

Die Bekanntmachung gibt der Heeres- und Marineverwaltung noch ein weitgehendes Recht über die Akteneinsicht. Nach dem geltenden Patentgesetze konnte auch die Heeres- und Marineverwaltung nicht über das Vorliegen einer Anmeldung und über deren Inhalt Kenntnis erhalten, falls diese Anmeldung nicht bekanntgemacht worden war. Nach \& 2 Absatz 1 der Bekanntmachung hat die Heeres- und Marineverwaltung das Recht, die Akten über die Anmeldung von Erfindungen und Gebrauchsmustern, welche die Interessen der Landesverteidigung oder der Kriegswirtschaft berühren, einzusehen. Es ist ausdrücklich gesagt, da $B$ die Einsicht der Heeres- und Marineverwaltun $_{j}$ freisteht. Diese Bestimmung ist dahin auszulegen, daß der Anmelder kein Recht zu Widersprüchen gegen die Akteneinsicht besitzt, während auch bei den ausliegenden Anmeldungen oder erteilten $\mathbf{P a}$ tenten ein Widerspruchsrecht gegen die Einsichtnahme der nicht ausgelegten oder veröffentlichten Stücke bestand. Diese neue Befugnis der Heeres- und Marineverwaltung auf unbegrenzte Einsicht aller Patent- und Gebrauchsmusterakten ist selbstverständlich von weittragender Bedeutung. Mit dieser Bestimmung ist die Geheimhaltung von Anmeldungen, welche die Interessen der Landesverteidigung oder der Kriegswirtschaft für die Heeres- und Marineverwaltung berühren, für die Heeres- und Marineverwaltung aufgehoben. Andere Länder haben den betreffenden Verwaltungen bereits ein gleiches Recht eingeräumt.

Da die Bekanntmachung und Auslegung bei Geheimpatenten fortfällt, so besteht auch nicht die Möglichkeit, im Einspruche der Erteilung des Patentes entgegenzutreten. Die einzig gegebene Möglichkeit ist die Nichtigkeitsklage. Bei Gebrauchsmustern besteht ebenso wie bisher die vor den ordentlichen Gerichten anzustrengende Löschungsklage. Nach dem geltenden Patentgesetze muß die Nichtig keitsklage wegen Nichtneuheit und Mangel der Erfindung innerhalb 5 Jahren nach der Bekanntmachung der Patenterteilung angestrengt werden. Diese Fristbestimmung kann für die Geheimpatente nicht bestehen, da ja eine Bekanntmachung der Patenterteilung nicht stattfindet. Die Nichtigkeitsklage kann also während der ganzen Dauer der Geheimpatente angestrengt werden. Der Umstand, da $B$ die Akten der Geheimpatente nicht zugänglich sind, und daß über den Wortlaut des erteilten Patentes nichts zu erfahren ist, wird die Nichtigkeitsklage natürlich erschweren. Der Nichtigkeitskläger steht vor der Frage, ein Patent zu bekämpfen, dessen Inhalt er nicht kennt, sondern über dessen Inhalt er nur Vermutungen besitzen kann. Allerdings kann dem Nichtigkeitskläger mit Zustimmung der Hreresund Marineverwaltung die Einsicht in die Akten gewährt werden. Die erwähnte Strafbestimmung für die Verbreitung und unbefugte Beschaffung der Kenntnis des Akteninhaltes dürfte einem Mißbrauche der Nichtigkeitsklage vorbeugen. Das Patentamt hat es auch in der Hand, nach der Sachlage, wie zum Beispiel nach der Vertrauenswürdigkeit des Klägers, die Akteneinsicht zu gewühren oder abzulehnen. Es ist in einem solchen Falle unvermeidlich, daß die Person des Antragstellers für Annahme oder Ablehnung des Antrages mit in Erwägung gezogen wird.

Die Geheimhaltung der Geheimpatente ist nicht unbegrenzt. Wenn das Patentamt nach der Anhörung der Heeres- und Marineverwaltung die Geheimbaltung des Patentes oder des Gebrauchsmusters nicht mehr als erforderlich erachtet, so richtet sich das weitere Verfahren nach den allgemeinen gesetzlichen Vorschriften (\$ 3 der Bekanntmachung). Es erfolgt also dann die Drucklegung der Patentschrift und die Bekanntmachung der Erteilung. In einem derartigen Falle würde dann also eine fünfjährige Frist zur Anstrengung der Nichtigkeitsklage beginnen. Die Aufhebung der Geheimhaltung kann seitens des Patentamtes von selbst vorgenommen werden, wobei die Hecres- und Marineverwaltung angehört werden muß. Der Patentinhaber kann seinerseits auch einen Antrag stellen, die Geheimhaltung aufzuheben. Die Ablehnung dieses Antrages wäre nach $\$ 16$ des Patentgesetzes gebührenfrei und ohne bestimmte Fristbegrenzung durch die Beschwerde anzufechten. $\$ 26$ des Patentgesetzes kommt hierbei nicht in Betracht, weil das Patent ja bereits erteilt ist. 